\title{
Analysis of red blood cell parameters by Talbot-projected fringes
}

\author{
Shilpi Agarwal \\ Varun Kumar \\ Chandra Shakher
}




\title{
Analysis of red blood cell parameters by Talbot-projected fringes
}

\author{
Shilpi Agarwal, Varun Kumar, and Chandra Shakher \\ Indian Institute of Technology Delhi, Instrument Design Development Centre, Hauz Khas, New Delhi, India
}

\begin{abstract}
Red blood cell (RBC) anomalies are significant symptoms for identification of health disorders and several blood diseases, which involve the modification of the parameters and biophysical characteristics of such cells. The aim of this study is to measure the three-dimensional phase information of healthy RBCs and their parameters, such as cell diameter, thickness, and hemoglobin $(\mathrm{Hb})$ content, using Talbot-projected fringes. The Talbot image of linear grating is projected onto an RBC slide. The deformed grating lines due to the shape and refractive index of RBCs are recorded by a CCD camera through a $20 \times$ microscope objective. Hilbert transform is used to extract the phase image from the deformed projected grating lines. Experimentally calculated values of diameter $(8.2 \mu \mathrm{m})$, thickness $(2.7 \mu \mathrm{m})$, and $\mathrm{Hb}$ content $(28.7 \mathrm{pg} /$ cell) are well within the limits available in the literature. The proposed system is robust and user-friendly and performs the imaging of RBCs with high axial and lateral resolution $(2.19 \mu \mathrm{m})$. @ 2017 Society of Photo-Optical Instrumentation Engineers (SPIE) [DOI: 10.1117/1.JBO.22.10.106009]
\end{abstract}

Keywords: Talbot effect; self-imaging; red blood cell imaging; Hilbert transform.

Paper 170390R received Jun. 16, 2017; accepted for publication Sep. 22, 2017; published online Oct. 13, 2017.

\section{Introduction}

In recent decades, several optical quantitative phase imaging techniques have been developed for biological cells, including human red blood cell (RBC) imaging. These techniques are spatial phase-shifting (e.g., Ref. 1), Fourier phase microscopy (e.g., Refs. 2 and 3), Hilbert phase microscopy (e.g., Ref. 4), digital holographic microscopy (e.g., Ref. 5), diffraction phase microscopy (e.g., Ref. 6), etc. In these methods, coherent, monochromatic light source such as lasers are used to measure the properties of biological cells. Because different properties of the biological cells, for example, phase, refractive index, and dry mass, are wavelength-dependent, ${ }^{7,8}$ some different interferometric or microscopic techniques, such as spectroscopic phase microscopy (e.g., Ref. 9), spectroscopic diffraction phase microscopy (e.g., Ref. 10), quantitative dispersion microscopy (e.g., Ref. 11), quantitative phase spectroscopy (e.g., Ref. 12), and dynamic spectroscopic phase microscopy (e.g., Ref. 13), etc., have been developed. In these techniques, multiple wavelength lasers, color filter wheels, and a broadband source with acousto-optic tunable filters are used to filter the multiple wavelengths and to determine the wavelength-dependent properties of biological cells. However, all of these techniques are costly, require complicated setups to align, need more optical components, and involve many mechanical moving parts. Partially coherent light (temporally low-coherent) sources such as a white light source, ${ }^{14}$ superluminescent diode,${ }^{15}$ and light-emitting device ${ }^{8}$ are also used for biological cell imaging. Although these (spectrally broad band) sources reduce the unwanted speckle noise, they also require a dispersion compensation mechanism for the dispersion correction. Furthermore, it is not a good choice to use a spectrally broad light source if the object or medium has an inhomogeneous spectral response. ${ }^{16}$

*Address all correspondence to: Chandra Shakher, E-mail: cshakher@iddc.iitd ac.in
The fringe projection is a noncontact technique that can be performed either with coherent or incoherent light, by illuminating the object with a fringe pattern generated by a binary or sinusoidal grating. The advantages of using a binary fringe pattern are-(1) simple: the coding and decoding algorithms are very simple, (2) fast: because the processing algorithm is very simple, it can reach very fast processing speeds, and (3) robust: since only two levels are used, it is very robust to the noise. ${ }^{17}$ The projection of a light pattern with a regular structure is a noncontact technique and easy to implement for measuring the three-dimensional (3-D) profile of a biological cell, which is decoded from the deformed fringe pattern recorded on the object. ${ }^{18}$ The digital fringe projection technique based on the Lau effect, Moiré method, Talbot effect has been used for surface profiling and contouring of reflecting objects. ${ }^{17-20}$ If the Talbot image of grating is projected on the object, the surface information is depth encoded in a deformed fringe pattern by a carrier frequency corresponding to the grating frequency. ${ }^{21}$ This depth encoded shape information is obtained by the phase extraction and image processing techniques..$^{21-23}$ The Talbotprojected fringe technique has been used for profiling an object from the macroscopic $(0.5 \mathrm{~mm})^{22}$ to microscopic $(11 \mu \mathrm{m})^{21}$ scales. Rodriguez-Vera et al. ${ }^{21}$ demonstrated the 3 -D contouring of diffuse objects for shape measurement using Talbot fringe projection. Microscopic shape and deformation measurement, calibration of microtopographic measurement system, and vibration analysis at microscale, etc. have also been investigated by the Talbot fringe projection method. . $^{22,23}$

In this paper, the microscopic 3-D imaging of RBCs is demonstrated using the Talbot-projected fringes in transmission mode. The phase information is extracted by Hilbert transform (HT). HT is simple to implement, and phase information can be extracted from deformed fringe patterns with a phase measurement accuracy of $\lambda / 25 .^{24}$ It requires less computational time, as

1083-3668/2017/\$25.00 @ 2017 SPIE 
compared with other phase extraction methods, such as Fourier transform, phase-shifting, etc.

\section{Theory}

The imaging of a periodic object without any imaging device is known as the Talbot effect. ${ }^{25-27}$ For plane wave illumination of a grating, behind periodic objects, at a distance $Z_{T}=$ $\mathrm{ka}^{2} / \lambda(k=2,4,6,8, \ldots)$, Fresnel diffraction gives well-defined images of periodic objects, known as Fourier images or Talbot images/self-images, whereas the intermediate intensity distribution appearing between the Fourier image planes is a Fresnel image. ${ }^{28,29}$ If these Talbot fringes (self-image) are projected on an RBC slide, these fringes are deformed according to the surface profile and refractive index of RBC. ${ }^{30}$ These deformed Talbot-projected fringes or codified optical signals are recorded by the CCD camera and used to decodify or extract the phase information ${ }^{21}$ of RBCs.

The diffraction intensity distribution of the grating G1 at the Talbot plane, after passing through the object (RBCs) is given as $^{31,32}$

$I(x)=A(x)+B \cos \left[\frac{2 \pi m x}{a}+\phi(x)\right]$,

where $A(x)$ is the background intensity distribution, $B$ is a constant, $m$ is the grating order, $a$ is the period of grating, and $\phi$ is the phase delay due to the presence of transparent phase object (RBCs).

The object phase is extracted using HT. HT is a linear operator that takes a function and produces a function with a shift of $\pi / 2$ in the same spatial domain. The imaginary part of the analytical signal can be practically generated by passing the original signal through a "Hilbert transform." A real function and its Hilbert-transformed function are related to each other in such a way that they together create an analytical signal, which can be written with an amplitude and phase, and the derivative of the phase can be identified as the instantaneous frequency. ${ }^{4,32,33}$

The background intensity must be eliminated prior to the application of HT for retrieving the phase. To isolate the sinusoidal term and to eliminate the background intensity, Eq. (1) is highpass filtered in the spatial domain. The filtered image can be written as

$I^{\prime}(x)=B \cos \left[\frac{2 \pi m x}{a}+\phi(x)\right]$.

Now the HT of the filtered image is performed to get the complex analytical signal associated with the real function $I^{\prime}(x)$. The complex analytical signal $Z(x)$ is given by ${ }^{4,33}$

$Z(x)=\frac{1}{2}\left\{I^{\prime}(x)+i \mathrm{HT}\left[I^{\prime}(x)\right]\right\}$,

where HT is the Hilbert transform.

The phase associated with $Z(x)$ can be extracted as

$\Delta \phi=\arctan \left\{\frac{\mathrm{HT}\left[I^{\prime}(x)\right]}{I^{\prime}(x)}\right\}$.

The complex analytical signal $Z(x)$ exhibits rapid phase modulation with spatial frequency $1 / a$; thus, $\Delta \phi$ is strongly wrapped. Finally, the phase $\phi(x)$ associated with the presence of the object is extracted as $4,32,33$
$\phi(x)=\Delta \phi-q(x), \quad$ where $q(x)=\frac{2 \pi m}{a}$.

The wrapped phase, $\Delta \phi$, is unwrapped using the Goldstein phase unwrapping algorithm. ${ }^{34}$

\section{Experimental Setup}

Figure 1 shows the schematic diagram of the experimental setup. A light beam from a He-Ne laser of wavelength $632.8 \mathrm{~nm}$ is expanded using a $40 \times$ microscope objective $(\mathrm{NA}=0.65)$ by Melles Griot (model No. 160/017 40/0.65) and filtered by a pinhole of 5- $\mu \mathrm{m}$ diameter. A collimating lens of diameter $50 \mathrm{~mm}$ and focal length $250 \mathrm{~mm}$ is employed to illuminate the grating G1 (300 lines $/ \mathrm{mm}$ ) by a collimated beam. The RBC slide is placed at Talbot distance $\left(Z_{T}\right)$ from the grating $\mathrm{G} 1(1.62 \mathrm{~cm})$, so the Talbot-projected fringes (self-image of grating) lie on the RBC slide surface.

To increase the lateral resolution for RBC imaging, a microscope objective $(20 \times, \mathrm{NA}=0.40)$ is placed such that the $\mathrm{RBC}$ slide with Talbot-projected fringe image should be at the focus of the microscope objective. A CCD camera is placed at a distance of $3.24 \mathrm{~cm}$ from the microscope objective to capture the maximum field of view.

For preparing a blood slide for RBC imaging (RBC slide), the blood samples were collected from the Indian Institute of Technology (IIT) Delhi, Hospital Pathology Lab, and approved by the responsible ethics committee of IIT Delhi, Hospital. The blood is diluted by mixing $1 \mathrm{ml}$ of blood with $10 \mathrm{ml}$ of saline solution $(\mathrm{NaCl})$ in a test tube. One drop of this diluted blood is placed at one end of a slide and is dispersed using another slide over the slide's length. The aim is to get a region, called a monolayer, in which the cells are spaced far enough apart to be differentiated.

When a prepared RBC slide is inserted at the Talbot plane, the Talbot-projected fringes get distorted. These distorted Talbot-projected fringes, having the phase, refractive index, and thickness information of RBCs, are recorded by a CCD camera (Make-Lumenera Corporation, Model-Infinity3$1 \mathrm{M})$. The pixel size on the CCD sensor is $6.45 \mu \mathrm{m} \times 6.45 \mu \mathrm{m}$. The total number of pixels is $1392 \times 1040$, and the sensor chip dimension is $2 / 3^{\prime \prime}$.

\section{Results}

HT is used for the evaluation of the phase map from the captured image.

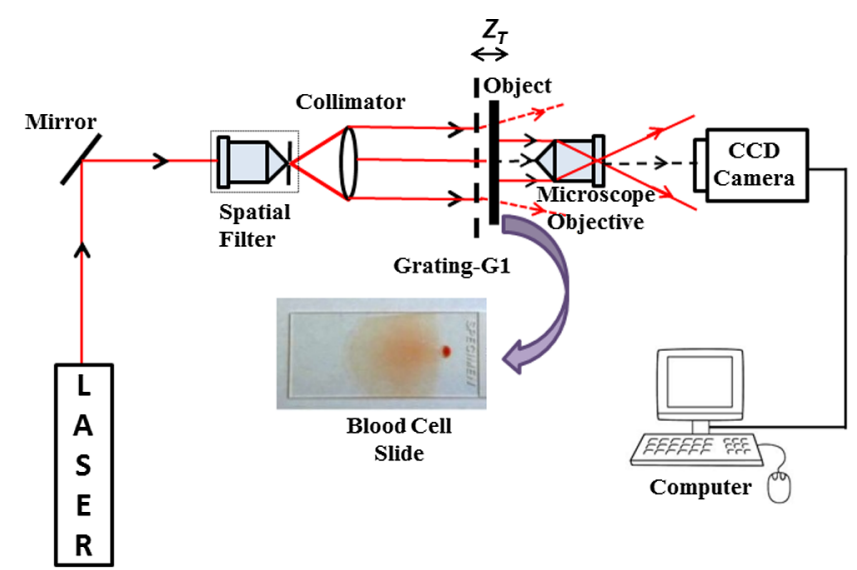

Fig. 1 Schematic diagram of the experimental setup. 


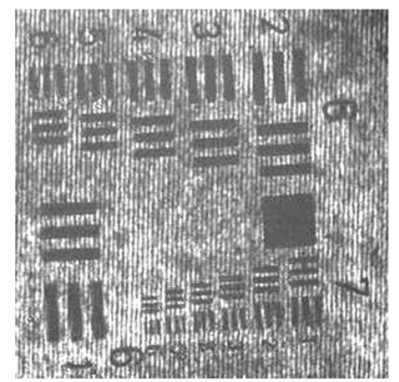

(a)

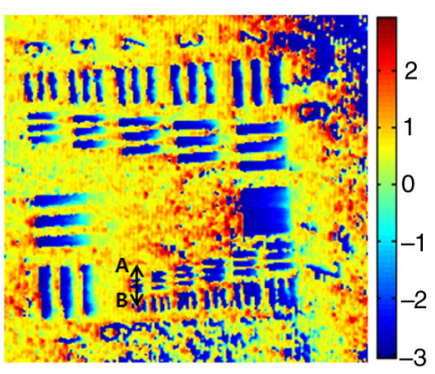

(b)

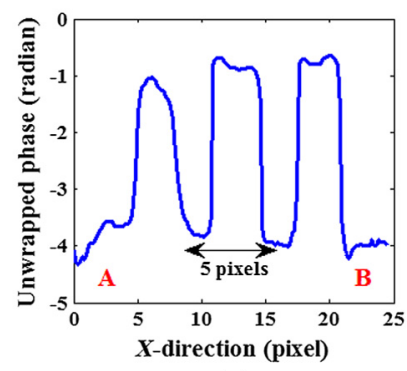

(c)
Fig. 2 USAF resolution test chart. (a) Recorded image, (b) reconstructed wrapped phase image, and (c) line profile along line $A B$ on unwrapped phase image.

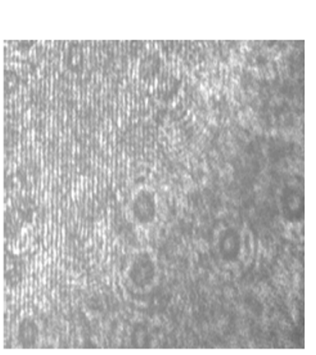

(a)

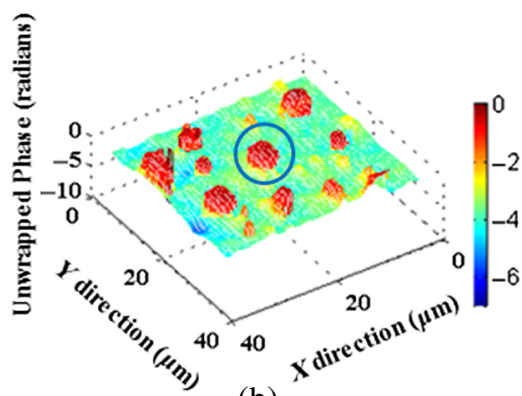

(b)
Fig. 3 Polystyrene beads. (a) Recorded image and (b) 3-D unwrapped phase.

Initially, the experiment is carried out using a USAF resolution test chart and polystyrene beads to know the resolution of the setup. A small portion of the USAF chart is captured by a CCD camera using Talbot-projected fringes and a self-imaging effect to know the lateral resolution of setup. No other imaging optics are used for recording the image. Figure 2(a) shows the recorded image of the USAF resolution test chart with Talbot-projected fringes. The phase image of the USAF resolution test chart is reconstructed using HT. From the reconstructed wrapped phase image of the test chart, the sixth element of the seventh group can be easily seen in Fig. 2(b). This element corresponds to 228.1 line pairs $/ \mathrm{mm}$ (i.e., the width of one line is $2.19 \mu \mathrm{m}$ in the USAF resolution test target). From the line profile of the unwrapped phase image shown in Fig. 2(c), drawn along line $\mathrm{AB}$ marked in the Fig. 2(b), the observed value of one pixel is $\sim 0.4 \mu \mathrm{m}$, which is used for the determination of the lateral dimension of object RBCs under study.

To study the accuracy of the system in retrieving the phase map and profiles, measurements are performed on polystyrene microbeads of a known diameter of $10 \mu \mathrm{m}$. The polystyrene beads are diluted in an index matching oil medium and placed on the glass slide to prepare the polystyrene beads sample slide. Figure 3(a) shows the recorded image of polystyrene beads, and Fig. 3(b) shows the reconstructed 3-D unwrapped phase corresponding to Fig. 3(a).

From the 3-D unwrapped phase map shown in Fig. 3(b), the phase of selected polystyrene bead is $3.8 \mathrm{rad}$. The size of the polystyrene bead is calculated by $\phi(x)=(2 \pi / \lambda)\left(n_{p}-n_{m}\right) d$, where $\phi(x)$ is the phase in radian, $d$ is the size of the polystyrene bead, $n_{p}$ is the refractive index of the polystyrene bead (1.59), and $n_{m}$ is the refractive index of the index matching oil medium (1.55).

The calculated size of the polystyrene bead is $9.57 \mu \mathrm{m}$. The size of the polystyrene bead, according to the specification by the supplier $(10 \mu \mathrm{m})$ is closely related to the calculated value.

Figure 4(a) shows the 3-D thickness map of a polystyrene bead, and Fig. 4(b) shows the thickness profile along line $\mathrm{AB}$ as shown in Fig. 4(a).

To check the consistency of the results, the same procedure was repeated with various polystyrene beads of the same size. Figure 5 shows the unwrapped phase map and the calculated thickness profile of selected polystyrene beads. The calculated size of different polystyrene beads as shown in Fig. 5 is 10.9, 9.4, 10.3, 10.1, and $9.7 \mu \mathrm{m}$. The experimentally calculated size of polystyrene beads shows a standard deviation (SD) of $0.32 \mu \mathrm{m}$ from the mean value of $9.96 \mu \mathrm{m}$. The results are quite consistent with the size of these beads given by the supplier. The percentage error between the experimentally calculated size of polystyrene beads (average size $9.96 \mu \mathrm{m}$ with SD 0.32 ) and the supplier specification $(10 \mu \mathrm{m})$ is $0.4 \%$.

A preliminary experiment is conducted on RBCs to ascertain the phase map and the viability of this technique for biological

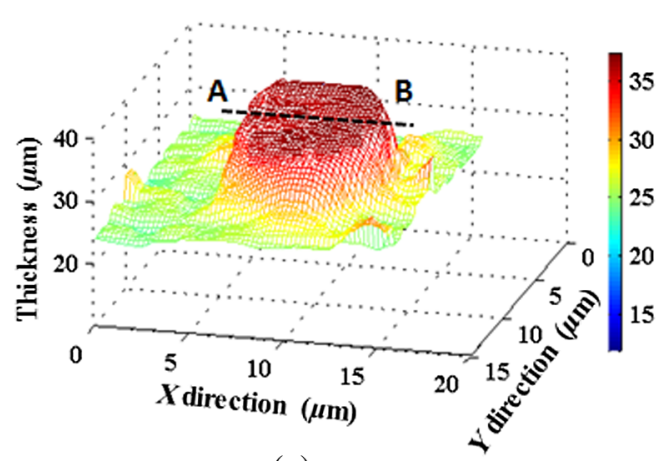

(a)

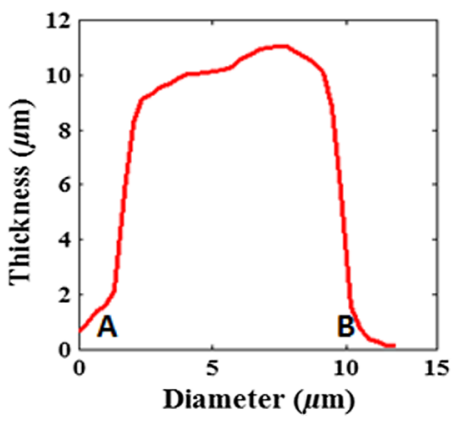

(b)

Fig. 4 (a) 3-D thickness map of polystyrene bead and (b) thickness profile along line AB. 


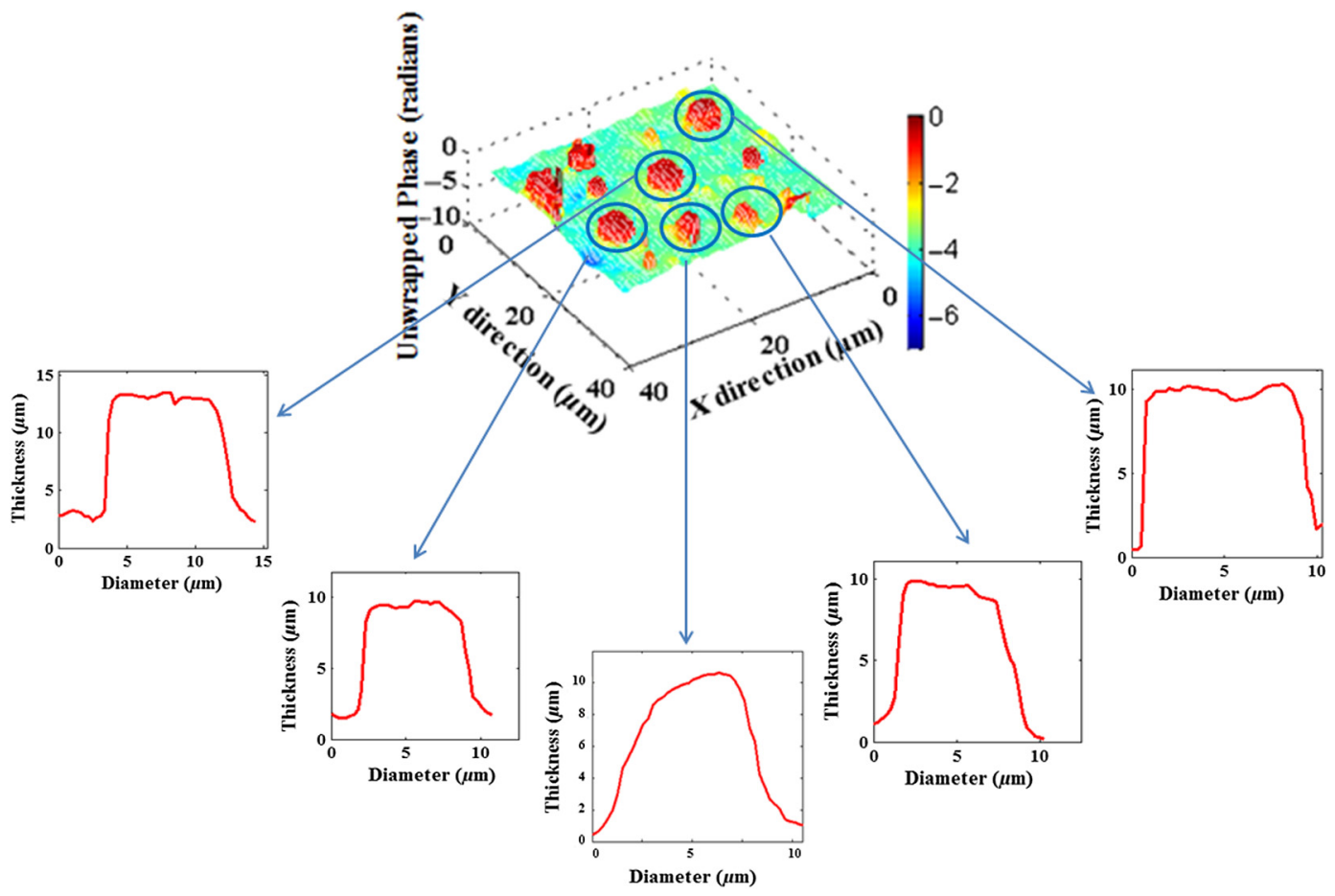

Fig. 5 3-D unwrapped phase map and the calculated thickness profile corresponding to selected beads.

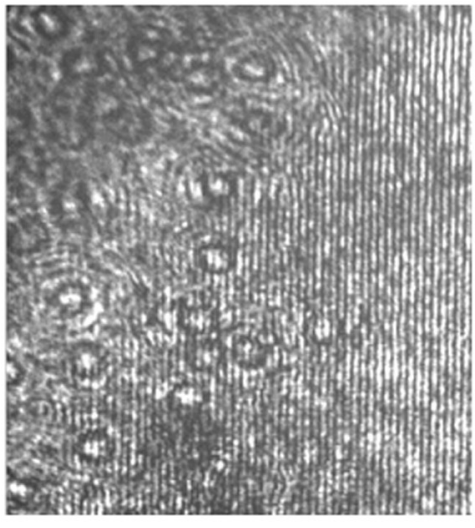

(a)

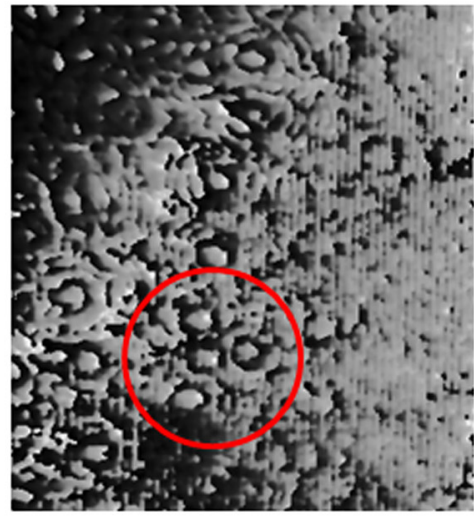

(b)

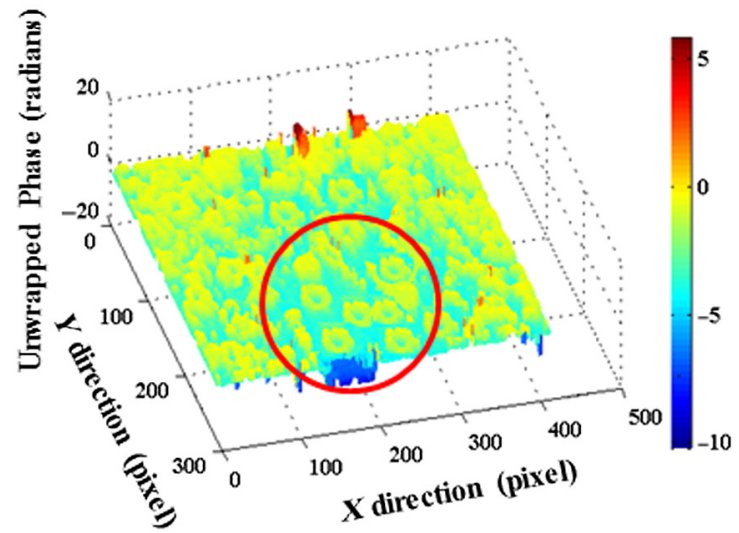

(c)

Fig. 6 (a) Recorded image, (b) 2-D wrapped phase of RBC, and (c) 3-D unwrapped phase of RBC.

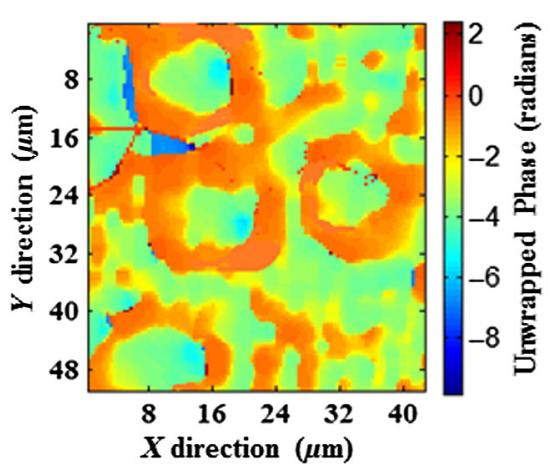

(a)

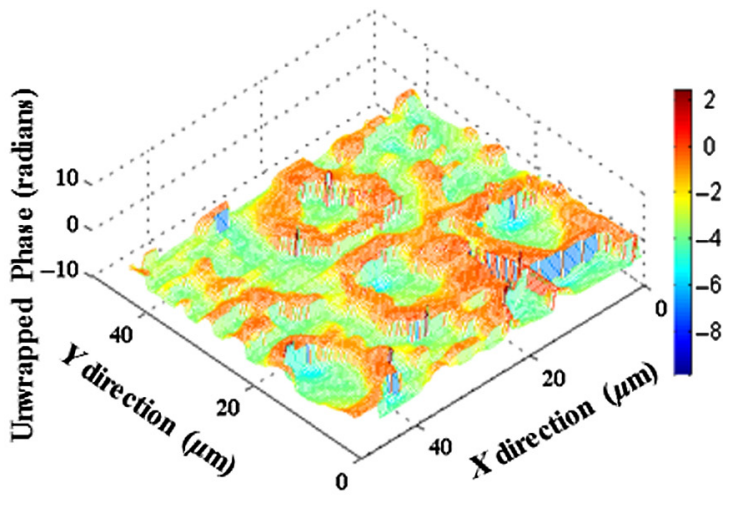

(b)

Fig. 7 Phase image: (a) 2-D unwrapped phase of selected portion and (b) 3-D unwrapped phase of RBC. 
cells. Figure 6(a) shows the recorded image of the RBCs. The phase information is obtained by the application of HT using Eq. (4). Figure 6(b) shows the wrapped phase, and Fig. 6(c) shows the 3-D unwrapped phase map corresponding to
Fig. 6(a). The obtained phase map is wrapped between 0 and $2 \pi$. This $2 \pi$ phase discontinuity is removed using the Goldstein phase unwrapping algorithm. ${ }^{34}$ Figures 7(a) and 7(b) show the two-dimensional (2-D) unwrapped phase map and 3-D

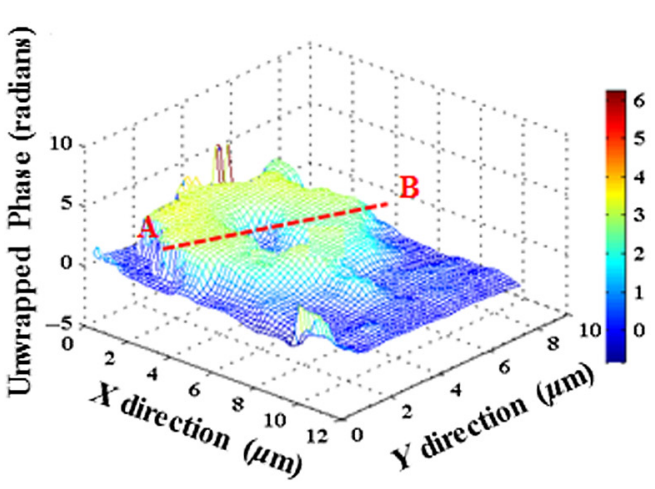

(a)

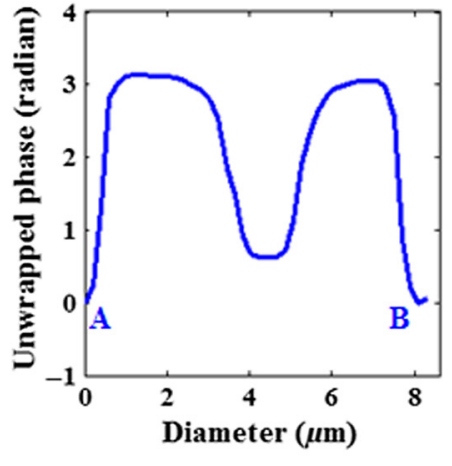

(b)

Fig. 8 (a) 3-D unwrapped phase of single RBC and (b) 2-D unwrapped phase profile.
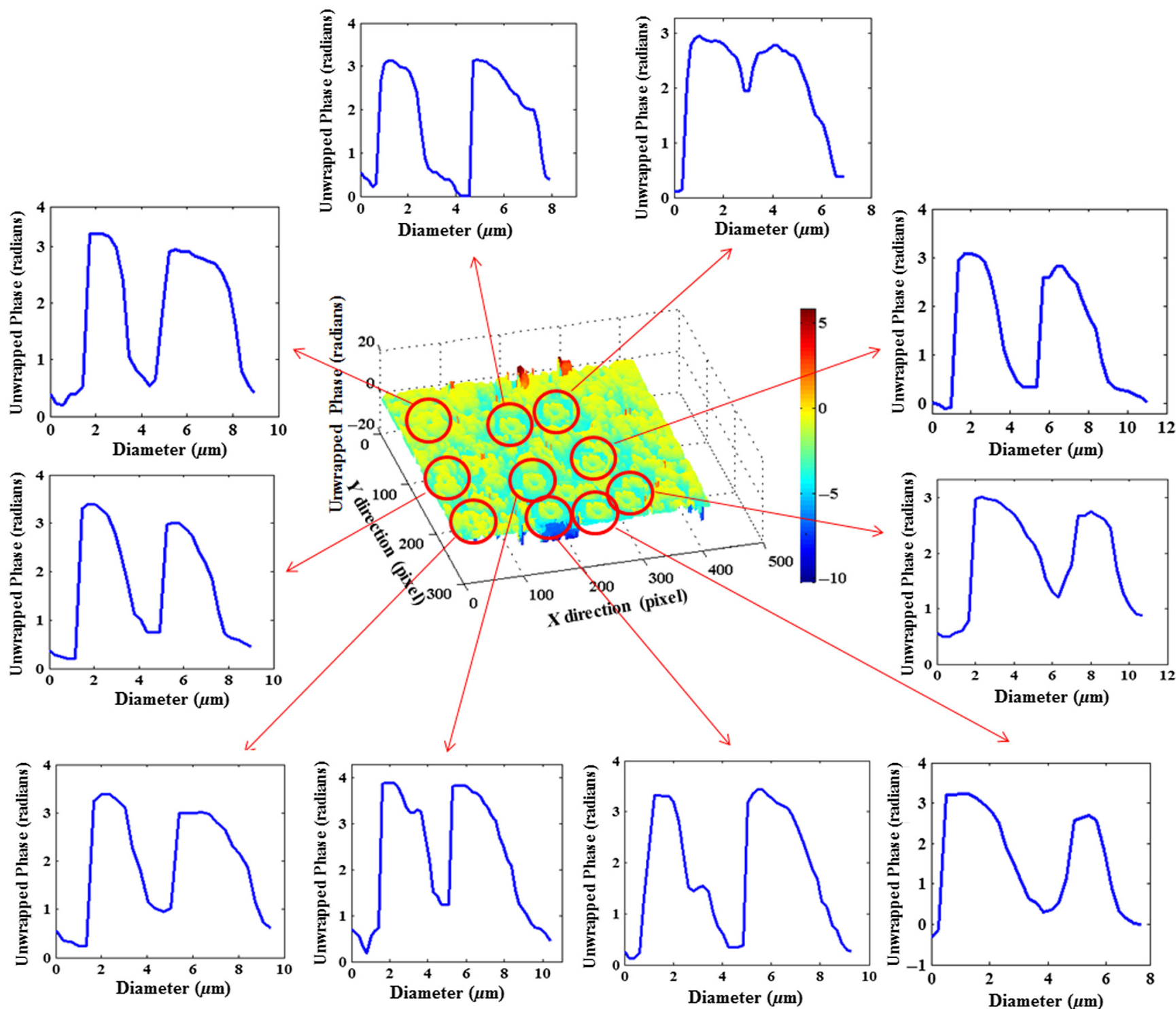

Fig. 9 3-D unwrapped phase map and obtained diameter of RBCs.

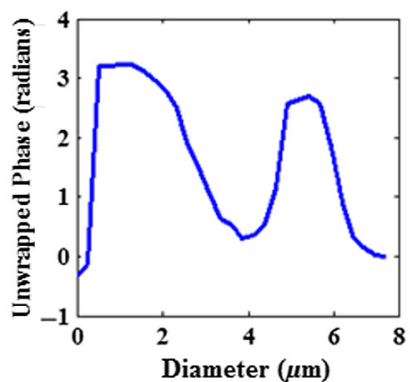


unwrapped phase map of selected RBCs from Fig. 6(b) (shown in circle), respectively.

Figure 8(a) shows the unwrapped phase map of a single RBC selected from Fig. 7(b), and Fig. 8(b) shows the phase profile with respect to position in $\mu \mathrm{m}$ (RBC diameter), obtained by drawing a line profile on phase map along line $\mathrm{AB}$. The experimentally calculated value of the selected RBC diameter is $\approx 8.2 \mu \mathrm{m}$.

The experiment was repeated for several RBCs. The unwrapped phase map of RBCs as shown in Fig. 6(c) was used to calculate the diameter of various RBCs. Figure (9) shows the unwrapped phase map and diameter of RBCs obtained by drawing a line profile on the phase image of various selected RBCs. The calculated diameter of different RBCs as shown in Fig. 9 is 6.8, 7.3, 8.1, 8.3, and $7.8 \mu \mathrm{m}$.

The mean value of the experimentally calculated diameter is found to be $7.6 \mu \mathrm{m}$ with SD $1.21 \mu \mathrm{m}$, which is in good agreement with the value available in the literature. ${ }^{35,36}$

The phase information retrieved by the proposed method can be expeditiously translated into thickness information, which in turn can be utilized to measure other relevant morphological parameters, such as cell shape and volume. As we know, the refractive index of biological cells is a vital parameter for determining the thickness and hemoglobin $(\mathrm{Hb})$ concentration in RBC. The thickness of the human RBC can be calculated using the following equation:

$t=\frac{\lambda}{2 \pi\left(n_{\mathrm{RBC}}-n_{m}\right)} \phi(x)$, where $\lambda$ is the wavelength of laser light, $\phi(x)$ is the optical phase, $n_{\mathrm{RBC}}$ is the refractive index of a healthy $\mathrm{RBC}, n_{m}$ is the refractive index of medium, and $t$ is the geometrical thickness of RBC. The refractive index of saline water for $\mathrm{He}-\mathrm{Ne}$ laser wavelengths $(632.8 \mathrm{~nm})$ is 1.331 , and the value of the refractive index of a healthy $\mathrm{RBC}$ is 1.394 , as reported in the literature. $^{37,38}$ The calculated value of the RBC thickness is $\approx 2.7 \mu \mathrm{m}$, which is in good agreement with the value available in the literature. ${ }^{35,39}$ Figure 10 (a) shows the 3-D thickness map of RBC, and Fig. 10(b) shows the thickness profile along line $\mathrm{AB}$ as shown in Fig. 10(a).

$\mathrm{Hb}$ content measures the amount of protein present in one RBC. For the measurement of the $\mathrm{Hb}$ content, the measured 2-D phase at the normal angle was used. The $\mathrm{Hb}$ content of an $\mathrm{RBC}$ is given as ${ }^{40,41}$

Hb content $=\frac{\lambda}{2 \pi \alpha} \sum \phi(x)$,

where $\alpha$ is the refractive index increment (assuming average $\alpha=0.2 \mathrm{ml} / \mathrm{g})^{40-42}$ and $\sum \phi(x)$ is the 2-D optical phase integrated over the entire cell area. The term $\sum \phi(x)$ is calculated by multiplying the phase $\phi(x)$ to the projected cell area (shape of the RBC is considered circular), so fewer errors are involved in assessing the phase value over a small area. ${ }^{40}$ The calculated mean value of the $\mathrm{Hb}$ content is $\sim 28.7 \mathrm{pg} /$ cell. $^{40,41}$ Figure 11 shows the 2-D and 3-D Hb contents of one RBC.

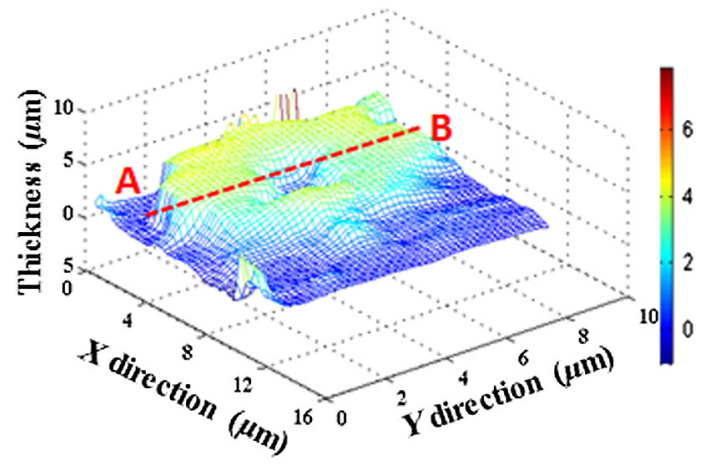

(a)

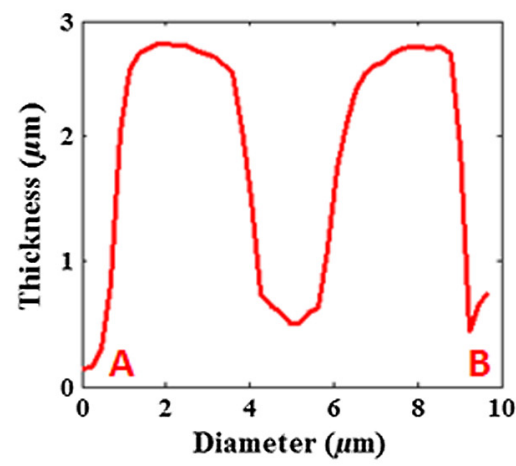

(b)

Fig. 10 (a) 3-D thickness map of $R B C$ and (b) thickness profile along line $A B$.

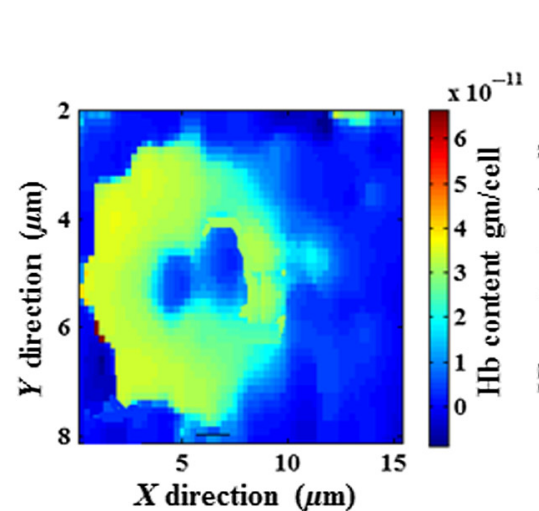

(a)

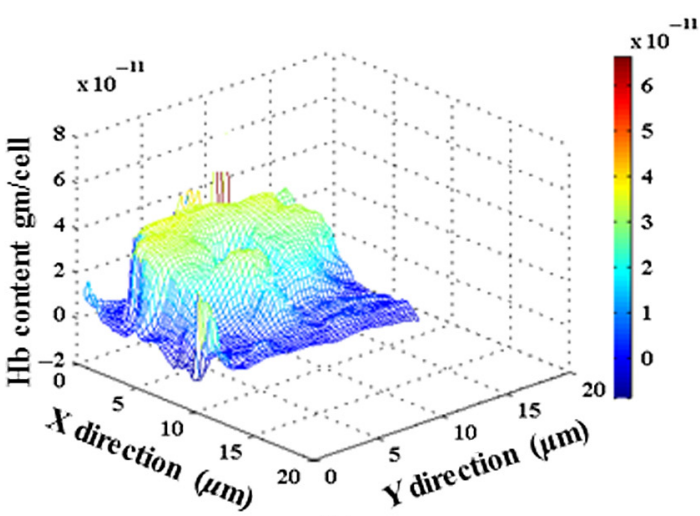

(b)

Fig. 11 Hemoglobin content of RBC: (a) 2-D and (b) 3-D. 
In addition, the $\mathrm{Hb}$ concentration in an $\mathrm{RBC}$ can also be obtained from the $\mathrm{Hb}$ content divided by the cellular volume.

\section{Conclusion}

We have presented a simple and low-cost, quantitative phase measurement technique for 3-D microscopic imaging of RBCs using the Talbot-projected fringes of a linear grating. The present method will open possibilities for diagnosing diseases as well as studying the pathophysiology of human RBCs. In addition, the stability of the proposed system from environmental perturbation due to its common-path geometry as well as its compactness and portability give it a high potential for clinical applications.

\section{Disclosures}

Shilpi Agarwal, Dr. Varun Kumar, and Professor Chandra Shakher report grants from Science and Engineering Research Board (SERB), Department of Science and Technology, Government of India, during the conduct of the study. The authors have no other potential conflicts of interest to disclose.

\section{Acknowledgments}

The financial assistance from the Science and Engineering Research Board (SERB), Department of Science and Technology, Government of India, under the project Sanction Order No. SR/S2/LOP-34/2013 and dated 20 October 2014, is highly acknowledged. The authors gratefully acknowledge the comments made by the referees to improve the paper.

\section{References}

1. S. K. Debnath and Y. Park, "Real-time quantitative phase imaging with a spatial phase-shifting algorithm," Opt. Lett. 36(23), 4677-4679 (2011).

2. G. Popescu et al., "Fourier phase microscopy for investigation of biological structures and dynamics," Opt. Lett. 29(21), 2503-2505 (2004).

3. N. Lue et al., "Quantitative phase imaging of live cells using fast Fourier phase microscopy," Appl. Opt. 46(10), 1836-1842 (2007).

4. T. Ikeda et al., "Hilbert phase microscopy for investigating fast dynamics in transparent systems," Opt. Lett. 30(10), 1165-1167 (2005).

5. B. Rappaz et al., "Measurement of the integral refractive index and dynamic cell morphometry of living cells with digital holographic microscopy," Opt. Express 13(23), 9361-9373 (2005).

6. G. Popescu et al., "Diffraction phase microscopy for quantifying cell structure and dynamics," Opt. Lett. 31(6), 775-777 (2006).

7. K. G. Phillips, L. J. Steven, and O. J. T. McCarty, "Measurement of single cell refractive index, dry mass, volume, and density using a transillumination microscope," Phys. Rev. Lett. 109(11), 118105 (2012).

8. V. Dubey et al., "Multispectral quantitative phase imaging of human red blood cells using inexpensive narrowband multicolor LEDs," Appl. Opt. 55(10), 2521-2525 (2016).

9. Y. Park et al., "Spectroscopic phase microscopy for quantifying hemoglobin concentrations in intact red blood cells," Opt. Lett. 34(23), 3668-3670 (2009).

10. B. Bhaduri et al., "Diffraction phase microscopy with white light," Opt. Lett. 37(6), 1094-1096 (2012).

11. D. Fu et al., "Quantitative dispersion microscopy," Biomed. Opt. Express 1(2), 347-353 (2010).

12. M. Rinehart, Y. Zhu, and A. Wax, "Quantitative phase spectroscopy," Biomed. Opt. Express 3(5), 958-965 (2012).

13. Y. Jang, J. Jang, and Y. K. Park, "Dynamic spectroscopic phase microscopy for quantifying hemoglobin concentration and dynamic membrane fluctuation in red blood cells," Opt. Express 20(9), 9673-9681 (2012).

14. B. Bhaduri, K. Tangella, and G. Popescu, "Fourier phase microscopy with white light," Biomed. Opt. Express 4(8), 1434-1441 (2013).

15. B. Kemper et al., "Characterisation of light emitting diodes (LEDs) for application in digital holographic microscopy for inspection of micro and nanostructured surfaces," Opt. Lasers Eng. 46(7), 499-507 (2008).
16. J. Rosen and M. Takeda, "Longitudinal spatial coherence applied for surface profilometry," Appl. Opt. 39(23), 4107-4111 (2000).

17. A. Nava-Vega et al., "Phase map by fringe projection with dammann gratings: an application to measure small objects," Rev. Mex. Fis. 61(5), 330-337 (2015).

18. S. Zhang, "Recent progresses on real-time 3D shape measurement using digital fringe projection techniques," Opt. Laser Eng. 48(2), 149-158 (2010).

19. M. Thakur, C. Quan, and C. J. Tay, "Surface profiling using fringe projection technique based on Lau effect," Opt. Laser Technol. 39(3), 453-459 (2007).

20. K. Creath and J. C. Wyant, "Moiré and fringe projection techniques," in Optical Shop Testing, 2nd ed., D. Malacara, Ed., pp. 653-685, Wiley, Hoboken, New Jersey (1992).

21. R. Rodriguez-Vera, D. Kerr, and F. Mendoza-Santoyo, "3-D contouring of diffuse objects by Talbot-projected fringes," J. Mod. Opt. 38(10), 1935-1945 (1991).

22. R. Rodriguez-Vera et al., "Calibration of a micro-topographic measurement system based on Talbot fringe projection," in Proc. 2007 SEM Annual Conf. and Exposition on Experimental and Applied Mechanics, Paper, Vol. 83 (2007).

23. R. Rodriguez-Vera et al., "Vibration analysis at microscale by Talbot fringe projection method," Strain 45(3), 249-258 (2009).

24. R. Onodera, H. Watanabe, and Y. Ishii, "Interferometric phase-measurement using a one-dimensional discrete Hilbert transform," Opt. Rev. 12(1), 29-36 (2005).

25. H. F. Talbot, "Facts relating to optical science. No. IV," J. Philos. Mag. Ser. 3 9, 401-407 (1836).

26. O. Bryngdahl, "Image formation using self-imaging techniques," J. Opt. Soc. Am. 63(4), 416-419 (1973).

27. K. Patorski, "The self-imaging phenomenon and its applications," in Progress in Optics, E. Wolf, Ed., Vol. 27, pp. 1-108, Elsevier Science Publishers B.V., North Holland, Amsterdam (1989).

28. J. M. Cowley and A. F. Moodie, "Fourier images: I-The point source," Proc. Phys. Soc. London Sec. B 70(5), 486-496 (1957).

29. J. T. Winthrop and C. R. Worthington, "Theory of Fresnel images. I. Plane periodic objects in monochromatic light," J. Opt. Soc. Am. 55(4), 373-381 (1965).

30. S. Agarwal, V. Kumar, and C. Shakher, "Cell imaging by Talbot projected fringes," in Imaging Systems and Applications, Paper ITh1E-3, Optical Society of America (2017).

31. Y. Takeda et al., "X-ray phase imaging with single phase grating," Jpn. J. Appl. Phys. 46, L89-L91 (2007).

32. N. Silin, S. Shin, and Y. Yu, "Three dimensional measurements by deflectometry and double Hilbert transform," J. Korean Phys. Soc. 69(3), 286-290 (2016).

33. X. Liang et al., "Single-shot slightly-off-axis interferometry based Hilbert phase microscopy of red blood cells," Biomed. Opt. Express 2(4), 987-995 (2011).

34. R. M. Goldstein, H. A. Zebker, and C. L. Werner, "Satellite radar interferometry: two-dimensional phase unwrapping," Radio Sci. 23(4), 713-720 (1988).

35. M. L. Turgeon, Clinical Hematology: Theory and Procedures, Lippincott Williams \& Wilkins, Philadelphia, Pennsylvania (2005).

36. E. L. Persons, "Studies on red blood cell diameter: III. The relative diameter of immature (reticulocytes) and adult red blood cells in health and anemia, especially in pernicious anemia," J. Clin. Invest. 7(4), 615-629 (1929).

37. I. Shock et al., "Optical phase nanoscopy in red blood cells using low-coherence spectroscopy," J. Biomed. Opt. 17(10), 101509 (2012).

38. P. M. S. Roma et al., "Total three-dimensional imaging of phase objects using defocusing microscopy: application to red blood cells," Appl. Phys. Lett. 104(25), 251107 (2014).

39. W. Jin et al., "Simulation of simultaneous measurement for red blood cell thickness and refractive index," Opt. Lasers Eng. 50(2), 154-158 (2012).

40. R. Barer, "Interference microscopy and mass determination," Nature 169, 366-367 (1952).

41. Y. Jang, J. Jang, and Y. Park, "Dynamic spectroscopic phase microscopy for quantifying hemoglobin concentration and dynamic membrane fluctuation in red blood cells," Opt. Express 20(9), 9673-9681 (2012).

42. H. Park et al., "3-D refractive index tomograms and deformability of individual human red blood cells from cord blood of newborn infants and maternal blood," J. Biomed. Opt. 20(11), 111208 (2015). 
Shilpi Agarwal received her MSc in physics with a specialization in advanced electronics from A. P. S. University Rewa (M.P.) in 2005. She has worked on a project on the magneto-optical Kerr effect at the Indian Institute of Technology Delhi. She was an assistant professor at an engineering college, Greater Noida, from 2006 to 2012. Currently, she is pursuing her doctorate degree in the area of diffractive optical elements and digital holography.

Varun Kumar received his MTech degree in instrument technology and his $\mathrm{PhD}$ in optical engineering from the Indian Institute of Technology, New Delhi, India, in 2010 and 2016, respectively. His research interests include digital holography, digital holographic microscopy and speckle pattern interferometry, and Talbot interferometry.
Chandra Shakher is an emeritus professor with the Instrument Design Development Centre, at the Indian Institute of Technology, New Delhi. He received his MTech degree in applied optics from the Indian Institute of Technology, Delhi in 1976 and his PhD from the Indian Institute of Technology, Madras in 1980. He has handled a large number of research projects and industrial consultancies. His research interest includes holography interferometry, speckle metrology, image processing, fiber optic current sensors, optical coherence tomography, and laser based instrumentation. He has 105 publications in international journals and 73 in conference proceedings. $\mathrm{He}$ is a fellow of Indian National Academy of Engineering, a senior member of OSA, and a fellow of SPIE. 